\title{
EVALUATION OF CLINICAL EFFECTS AND TOLERABILITY OF CEFEPIME IN HOSPITAL SETTINGS IN BANGLADESH
}

\author{
ISLAM M ${ }^{1}$, RAHMAN ABMF 2 , DAS BC ${ }^{3}$, HOSSAIN MZ ${ }^{4}$, PERVIN $\mathrm{K}^{5}$
}

\begin{abstract}
Background: Cefepime a parenteral fourth generation cephalosporin is active against many Gram-positive and Gram-negative organisms. The study aimed to evaluate the effectiveness and tolerability of cefepime when used alone or in combination with other antibiotics for the treatment of infections in hospital settings in Bangladesh.
\end{abstract}

Methods: This multi-centric, prospective observational study enrolled eligible males and females $>18$ years who had been prescribe cefepime as a part of treatment strategy regardless of the purpose of hospitalization. No therapeutic intervention was implied rather treatment strategy and dosage of cefepime was on individual physician's discretion as per routine clinical practice. Percentage of patients who would have clinical success in terms of cure, improvement or failure was evaluated during the end of cefepime therapy. Patient's overall tolerability to cefepime therapy as excellent, good, satisfactory and poor was considered for safety assessment. The outcome analysis was done in patients' available follow-up at the end of therapy.

Results: A total of 1000 patients were enrolled in this study between March 2013 and September 2014 in different hospital settings of Internal Medicine, Coronary Care Unit, Orthopedic, Intensive Care Unit, Surgery and Nephrology (51.6\%, 17.8\%, 15.2\%, 11.6\%, 2.2\% \& 1.6\%). The mean $( \pm S D)$ age of patients was 50.1 ( \pm 14.7$)$ years (range 18-100) and $64.3 \%$ were males. Bacteriology or culture sensitivity report was available with 67 patients at baseline and most common microorganisms were E coli (35.8\%), Pseudomonas sp. (20.8\%), Klebsiella sp. (16.4\%), Staphylococcus sp. (11.9\%) and Streptococcus sp. (7.4\%) and others (7.4).

Cefepime was used as medical or surgical prophylaxis in $52.2 \%$ of the patients. In $47.8 \%$ of patients cefepime was prescribed for treatment of infections including lower respiratory tract infections, trauma / fracture related wound infections, urinary tract infections, treatment of gangrene and septicaemia $(39.7 \%, 31.8 \%, 10.9 \%, 9.2 \% \& 8.4 \%)$.

The dose of cefepime was $500 \mathrm{mg}$ to $2 \mathrm{gm}$ twice or thrice daily with the mean $( \pm S D)$ duration of 6.1 ( \pm 2.1 ) days (range 2-14). 55 patients were lost to follow-up at the end of therapy and 25 died due to congestive heart failure, renal or hepatic failure and multiple organ failure. On physicians end of therapy overall clinical outcome evaluation, $70.7 \%$ of the patients were cured, $24.9 \%$ improved and $4.4 \%$ had no improvement or worsening in the sign and symptoms. Overall tolerability of cefepime was good in $56.6 \%$, excellent in $28.7 \%$, satisfactory in $9.8 \%$ and poor in $4.9 \%$ of patients. No non-serious adverse event was reported in this study. Nonetheless, the 25 death cases would be considered as serious adverse events.

Conclusion: Cefepime therapy in different indications was effective in hospitalized patients for cure (67.5\%) or improvement (26.9\%) of sign and symptoms. It was also well tolerated in $85.3 \%$ of patients as assessed by the treating physicians

J Dhaka Med Coll. 2018; 27(1) : 17-24

1. Dr. Mohammad Manirul Islam, Assistant Professor Dept. of Haematology, Dhaka Medical College \& Hospital

2. Dr. ABM Fazlur Rahman, Head of the Dept. Orthopaedics, Dhaka National Medical College \& Hospital.

3. Dr. Bidhan C Das, Associate Professor Dept. of Surgery, Bangabondhu Sheikh Mujib Medical University

4. Dr. Mohammad Zaid Hossain, Associate Professor, Department of Medicine, Dhaka Medical College

5. Dr. Kumkum Pervin, Head of Pharmacovigilance (CSL), Sanofi Bangladesh Limited

Correspondence : Dr. Mohammad Manirul Islam, Assistant Professor Dept. Of Haematology, Dhaka Medical College \& Hospital. Mobile: 01715049573 E-mail: manirk54@yahoo.com

Received: 10 May 2017 Revision: 5 July 2017

DOI: http://dx.doi.org/10.3329/jdmc.v27i1.38890

Accepted: 01 September 2017 


\section{Introduction}

Proper and timely choice of the antibiotic therapy for the management of infection in hospitalized patients is an immense challenge to the clinicians. ${ }^{1}$ Antibiotic treatment is the sole or even major intervention in infectious disease management. Conversely, increasing resistance to the antibiotics poses the real problem of increased morbidity \& mortality, resulting in requirement of newer anti-bacterial options for managing critical conditions. In course of time the 4th generation cephalosporin - cefepime was introduced in Bangladesh, where managing hospital acquired pneumonia, septicemia, febrile neutropenia etc. are still a challenge.

Cefepime, a parenteral fourth-generation extended spectrum cephalosporin antibiotic active in vitro against a broad spectrum of gram-positive and gram-negative anaerobic bacteria. The gram-positive spectrum is similar to that of cefotaxime, the gram-negative spectrum is similar to that of ceftazidime, and many, though not all, organisms resistant to these two agents remain susceptible to cefepime, prompting the fourth-generation designation. ${ }^{2}$ Cefepime has a quaternary nitrogen that is positively charged at the 3 position, providing the properties of a zwitterion. A 2-aminothiazolyl-acetamido group in the side chain at the 7-position with an alpha-oxyimino substitution may enhance stability against beta-lactamases by preventing the enzymes' approach to the main nucleus. ${ }^{3}$ Cefepime has a high affinity for penicillinbinding proteins and due to its zwitterionic configuration, rapidly penetrates outermembrane porin channels of bacteria. ${ }^{2}$

Cefepime is stable against many of the common plasmid and chromosome mediated betalactamases and has a decreased propensity to induce beta-lactamases compared with other beta-lactam antibiotics. ${ }^{4}$ Clinical studies showed that cefepime is as effective as cefotaxime or ceftazidime in patients with infections of the lower respiratory tract, skin and skin structures, urinary tract or female reproductive system. ${ }^{3}$ Cefepime reduces fever as effectively as ceftazidime or piperacillin plus gentamicin in neutropenic patients. ${ }^{3}$

Cefepime is generally well tolerated, with a tolerability profile similar to those of other parenteral cephalosporins. In clinical trials, the majority of the adverse events (AE) experienced were mild to moderate and reversible. The most common adverse events with causal relationship to cefepime reported in clinical trials included rash and diarrhea. Other less common adverse events included pruritus, urticarial, nausea, vomiting, oral candidiasis, colitis, headache, fever, erythema and vaginitis. ${ }^{4}$

The effectiveness of cefepime is already established. However, data from Bangladesh are lacking on its effectiveness and tolerability when used alone or in combination with other antibiotics for the treatment of infections in hospital settings. This observational study aimed to collect such data in real-world settings in Bangladesh that would help to establish the profile of patients as well as indications of cefepime use.

\section{Methods}

This was a multi-centric, prospective observational study on evaluation of clinical effects and tolerability of cefepime for treating patients with infections in hospital settings between 19 March 2013 and 29 September 2014 by 35 physicians in Bangladesh. The sites or investigators were selected on convenience basis from a list of hospital based physicians available in company database. Patients aged above 18 years whose physician had decided to prescribe cefepime as a part of treatment strategy were included in the study. Physicians included eligible consecutive patients, upon informed consent, who met the inclusion criteria regardless of the purpose of hospitalization. The patients known to have had hypersensitivity to cefepime, cephalosporins, penicillins, or other beta-lactam antibiotics were excluded from study. 
Being observational study, dose and duration of cefepime therapy was on physician's discretion according to routine treatment strategy. There was no defined visit schedule for the patients but the physicians were asked to report updated patient data at the end of cefepime therapy. The physicians gathered patient data, on paper copy of Data Collection Forms (DCF), mainly on demographics, risk factors, co-morbidities, clinical diagnosis, information on laboratory investigations, treatment plan including dose and duration of cefepime therapy at baseline.

Primary end point of the study was aimed to the percentage of patients who would have clinical success in terms of clinically cured, improved or treatment failure in different indications where cefepime was prescribed [(i) Cure: Disappearance of all pre-treatment signs and symptoms of infection. (ii) Improvement: Improvement in, or partial disappearance of signs and symptoms without requiring further antibacterial therapy. (iii) Failure: No change in, or worsening of baseline signs and symptoms requiring modification of treatment, i.e. addition of or switch to another antibacterial therapy.] Demographics, types of infections, comorbidities, risk factors, relevant medical or surgical history, relevant medications, dose and duration of cefepime therapy were evaluated to assess clinical profile of the patients treated with cefepime in hospital settings. Overall tolerability of therapy (as excellent, good, satisfactory or poor) in patients was considered for safety assessment of cefepime therapy [(i) Excellent: with no adverse event reported, (ii) Good: with mild adverse event reported, (iii) Satisfactory: moderate adverse event reported requiring any interference, (iv) Poor: with serious adverse events (SAE) requiring interference].

Patients who completed follow-up visit at the end of antibiotic therapy were considered for analysis. Statistical analysis was mainly descriptive and was summarized as mean, median, standard deviation, minimum, maximum and percentages for continuous parameters and frequency and percentages for categorical parameters. Statistical analysis was done using SPSS 17.0.

\section{Results}

A total of 1000 patients (Figure 1) were enrolled in this study between March 2013 and September 2014 from different hospital settings namely from Internal Medicine (51.6\%), Coronary Care Unit (17.8\%), Orthopedic (15.2\%), Intensive Care Unit (11.6\%), Surgery (2.2\%) and Nephrology (1.6\%). The mean $( \pm \mathrm{SD})$ age of patients was $50.1( \pm 14.7)$ years (range 18-100). More than half (510, $51 \%)$ of the patients were aged 50 years and above and 77 (7.7\%) were below 30. 64.3\% (643) were males.

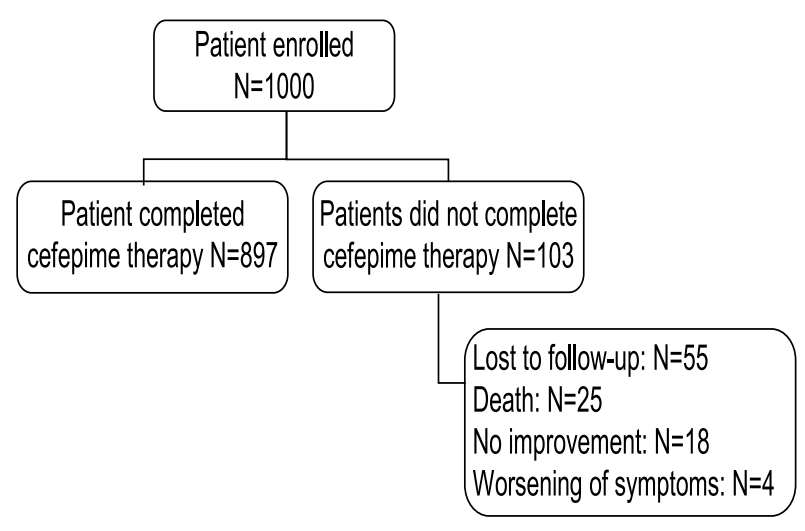

\section{Fig.-1: Patient flow chart}

Patients residing in urban were more $(42.6 \%)$ compared to semi-urban (34\%) and rural $(23.4 \%)$ areas. $81.1 \%(811)$ of the patients were unemployed and only $18.9 \%$ (189) were engaged in different professional works. Diabetes mellitus (51.9\%), COPD (28.6\%) and renal failure $(13.7 \%)$ were the most common associated comorbidities. They also had history of congestive heart failure $(6.3 \%)$, hypertension $(6.1 \%)$ and burn $(6.1 \%)$ injury. $4.5 \%$ of the patients was suffering from cancers and $10.1 \%$ had other medical conditions such as immunocompromised, hepatic failure, neurological diseases and multi-organ failure. Patient demographic characteristics and associated comorbidities are listed in Table 1. 
Table-1

Baseline demographics and clinical characteristics of study patients

\begin{tabular}{|c|c|c|}
\hline & $\begin{array}{c}\text { Frequency } \\
(\mathrm{n}=1000)\end{array}$ & $\begin{array}{c}\text { Percentage } \\
(\%)\end{array}$ \\
\hline \multicolumn{3}{|c|}{ Demographics (mean \pm SD) } \\
\hline Age (mean $\pm \mathrm{SD}$ ) yrs & $50.1 \pm 14.7$ & \\
\hline \multicolumn{3}{|l|}{ Gender } \\
\hline Male & 643 & 64.3 \\
\hline Female & 357 & 35.7 \\
\hline \multicolumn{3}{|l|}{ Locality } \\
\hline Urban & 426 & 42.6 \\
\hline Semi-urban & 340 & 34 \\
\hline Rural & 234 & 23.4 \\
\hline \multicolumn{3}{|l|}{ Profession } \\
\hline Unemployed & 811 & 81.1 \\
\hline Professional & 189 & 18.9 \\
\hline \multicolumn{3}{|l|}{ Smoking habit } \\
\hline Non-smoker & 719 & 71.9 \\
\hline Smoker & 281 & 28.1 \\
\hline \multicolumn{3}{|l|}{ Hospital settings } \\
\hline $\mathrm{CCU}$ & 178 & 17.8 \\
\hline ICU & 116 & 11.6 \\
\hline Medicine & 516 & 51.6 \\
\hline Orthopedic & 152 & 15.2 \\
\hline Surgery & 22 & 2.2 \\
\hline Nephrology & 16 & 1.6 \\
\hline \multicolumn{3}{|c|}{ Associated comorbidities in patients } \\
\hline Diabetes Mellitus & 519 & 51.9 \\
\hline COPD & 286 & 28.6 \\
\hline Renal failure & 137 & 13.7 \\
\hline Congestive Heart & 63 & 6.3 \\
\hline \multicolumn{3}{|l|}{ Failure (CHF) } \\
\hline Burns & 61 & 6.1 \\
\hline Hypertension (HTN) & 61 & 6.1 \\
\hline Cancer & 45 & 4.5 \\
\hline $\begin{array}{l}\text { Other medical condition } \\
\text { (immunocompromised, } \\
\text { hepatic failure, neurol } \\
\text { diseases, MOF etc.) }\end{array}$ & 101 & 10.1 \\
\hline $\begin{array}{l}\text { No related diseases / } \\
\text { risk factors }\end{array}$ & $156 \quad 15.6$ & \\
\hline
\end{tabular}

All of the patients were prescribed injection cefepime as per routine treatment strategy. In $52.2 \%$ of the patients cefepime therapy was empiric as medical or surgical prophylaxis though data about medical condition and surgical procedure was not recorded. The physicians also prescribed cefepime for treatment of infections in $47.8 \%$ of patients including lower respiratory tract infections (39.7\%), trauma/fracture related wound infections $(31.8 \%)$, urinary tract infections $(10.9 \%)$, treatment of gangrene $(9.2 \%)$ and septicaemia (8.4\%) (Table 2). In 247 patients cefepime was prescribed along with other antibiotics included levofloxacin $(41.7 \%)$, flucloxacillin $(20.2 \%)$, metronidazole $(11.3 \%)$, amikacin (10.9\%) and clindamycin (8.1\%) (Table $3)$.

\section{Table-II}

Indications of cefepime use in hospitalized patients

\begin{tabular}{lcc}
\hline $\begin{array}{l}\text { Clinical indications } \\
\text { of cefepime use }\end{array}$ & $\begin{array}{r}\text { Frequency } \\
(\mathrm{N}=1000)\end{array}$ & $\begin{array}{c}\text { Percent } \\
(\%)\end{array}$ \\
\hline Antibiotic Prophylaxis & 522 & 52.2 \\
Treatment of infections & 478 & 47.8 \\
LRTIs & 190 & 39.7 \\
Wound infections due to & 152 & 31.8 \\
trauma/fracture & & \\
UTIs & 52 & 10.9 \\
Gangrene & 44 & 9.2 \\
Septicaemia & 40 & 8.4 \\
\hline
\end{tabular}

Table-III

Other antibiotics prescribed along with cefepime in patients

\begin{tabular}{lcc}
\hline Other antibiotics & \multicolumn{2}{c}{ Patients $(\mathrm{N}=247)$} \\
\cline { 2 - 3 } & $\mathrm{N}$ & $\%$ \\
\hline Levofloxacin & 103 & 41.7 \\
Amikacin & 27 & 10.9 \\
Clindamycin & 20 & 8.1 \\
Flucloxacillin & 50 & 20.2 \\
Metronidazole & 28 & 11.3 \\
Others & 19 & 7.7 \\
\hline
\end{tabular}


Being observational study the dose and duration of cefepime therapy was upon physician's own discretion. However, the dose of cefepime was $500 \mathrm{mg}, 1 \mathrm{gm}$ or $2 \mathrm{gm}$ twice or thrice daily in $21.2 \%, 77.2 \%$ and $1.6 \%$ of patients respectively. The daily dose and frequency of cefepime injection used for prophylaxis and treatment of infection are shown Table 4a. Cefepime $1 \mathrm{gm}$ twice daily was the preferred dose of choice in majority $(47.5 \%)$ of the patients. The mean $( \pm \mathrm{SD})$ duration of cefepime therapy was $6.1( \pm 2.1)$ days which was varied from $2(1.7 \%)$ to 14 days $(2.1 \%)$. In majority of the patients $(73.6 \%)$ the duration of cefepime therapy was 5 to 7 days. However, $16.1 \%$ of the patients received therapy for less than 5 days and was continued for more than 7 days in $10.3 \%$ of patients. Table $4 \mathrm{~b}$ shows the duration of cefepime injection for prophylaxis and treatment of infections.

Among the patients 897 (89.7\%) completed cefepime therapy as prescribed. In the patients who (103) did not complete cefepime therapy, the main reason was lost to follow-up (55), death (25), lack of improvement (18) or worsening of symptoms (4) as shown in Table 5. None of the 25 death cases was related to cefepime therapy; rather the patients were suffering from congestive heart failure, renal or hepatic failure and multiple organ failure. The patients who were not available for follow-up were excluded from the outcome analysis at the end of therapy.

Table 4a

Cefepime dose and daily frequency in patients

\begin{tabular}{lcccc}
\hline $\begin{array}{l}\text { Use of cefepime } \\
\text { injection } \mathrm{N}=1000\end{array}$ & \multicolumn{2}{c}{ Daily Dose } & \multicolumn{2}{c}{ Daily Frequency } \\
\cline { 3 - 4 } & $\mathrm{N}(\%)$ & $\mathrm{N}(\%)$ \\
\hline Prophylaxis & $500 \mathrm{mg}$ & $119(22.8)$ & $105(88.2)$ & $14(11.8)$ \\
& $1 \mathrm{gm}$ & $395(75.7)$ & $296(74.9)$ & $99(25.1)$ \\
& $2 \mathrm{gm}$ & $8(1.5)$ & $2(25)$ & $6(75)$ \\
Treatment of infections & $500 \mathrm{mg}$ & $93(19.5)$ & $75(80.6)$ & $18(19.4)$ \\
& $1 \mathrm{gm}$ & $377(78.9)$ & $179(47.5)$ & $198(52.5)$ \\
& $2 \mathrm{gm}$ & $8(1.7)$ & $5(62.5)$ & $3(37.5)$ \\
\hline
\end{tabular}

Table 4b

Duration of cefepime therapy in patients

\begin{tabular}{lcccc}
\hline $\begin{array}{l}\text { Use of cefepime } \\
\text { injection }\end{array}$ & $\begin{array}{c}\text { Cefepime } \\
\text { Dose }\end{array}$ & \multicolumn{3}{c}{$\begin{array}{c}\text { Duration of therapy } \\
\end{array}$} \\
\cline { 2 - 5 } & & $<5$ days & $5-7$ days & $>7$ days \\
\hline Prophylaxis & $500 \mathrm{mg}$ & $40(33.6)$ & $68(57.1)$ & $11(9.2)$ \\
& $1 \mathrm{gm}$ & $86(21.8)$ & $282(71.4)$ & $27(6.8)$ \\
& $2 \mathrm{gm}$ & - & $6(75.0)$ & $2(25.0)$ \\
Treatment of infections & $500 \mathrm{mg}$ & $19(20.4)$ & $59(63.4)$ & $15(16.1)$ \\
& $1 \mathrm{gm}$ & $16(4.2)$ & $315(83.6)$ & $46(12.2)$ \\
& $2 \mathrm{gm}$ & - & $6(75.0)$ & $2(25.0)$ \\
\hline
\end{tabular}


Table-V

Reasons of discontinuation of cefepime therapy in patients

\begin{tabular}{lcc}
\hline & $\begin{array}{c}\text { Patients } \\
\text { (N=1000) } \\
\%\end{array}$ \\
\hline Lost to FU & 55 & 5.5 \\
No improvement & 18 & 1.8 \\
Worsening & 4 & 0.4 \\
Death & 25 & 2.5 \\
AE & 1 & 0.1 \\
\hline
\end{tabular}

The clinical outcome analysis was done in 945 patients whose data were available for end of therapy follow-up. According to the physicians overall clinical assessment in 454 patients (24 lost to follow-up) who were prescribed cefepime injection for treatment of infections, $70.7 \%$ of the patients were cured from the disease and $24.9 \%$ had improvement of sign and symptoms without requiring further antibiotic therapy. However, $4.4 \%$ of the patients had no improvement or worsening in the sign and symptoms. The 25 death cases were also included in treatment failure.

Among the patients with bacteriology report at the initiation of cefepime therapy data of 64 was available for end of therapy clinical outcome analysis. The highest $(60.0 \%)$ cure rate was observed in Streptococcal infections. In case of infections with Pseudomonas, Klebsiella, E.coli and Streptococcus and the cure rate was $53.8 \%$, $45.5 \%, 43.5 \%$ and $28.6 \%$ respectively. The overall clinical outcome is shown in Table $6 a$, $6 \mathrm{~b}, 6 \mathrm{c}$ and $6 \mathrm{~d}$. The overall clinical outcome is shown in Table 6a, 6b, 6c and 6d.

Table 6a

Physicians' assessment on overall clinical outcome of Cefepime in the patients

\begin{tabular}{lcc}
\hline Overall clinical outcome & \multicolumn{2}{c}{ Patients $(\mathrm{N}=454)$} \\
& $\mathrm{N}$ & $\%$ \\
\hline Cured & 321 & 70.1 \\
Improvement & 113 & 24.9 \\
Failure & 20 & 4.4 \\
\hline
\end{tabular}

Table 6b

Overall clinical outcome in patients with initial bacteriology report

\begin{tabular}{lcccc}
\hline & Cured & Improved & Failure & Total \\
& $\mathrm{N}(\%)$ & $\mathrm{N}(\%)$ & $\mathrm{N}(\%)$ & $\mathrm{N}=64$ \\
\hline E.coli & $10(43.5)$ & $11(47.8)$ & $2(8.7)$ & 23 \\
Klebsiella & $5(45.5)$ & $6(54.5)$ & - & 11 \\
Pseudomonas & $7(53.8)$ & $6(46.2)$ & - & 7 \\
Staphylococcus & $2(28.6)$ & $4(57.1)$ & $1(14.3)$ & 13 \\
Streptococcus & $3(60.0)$ & $1(20.0)$ & $1(20.0)$ & 5 \\
Others & $3(60.0)$ & $2(40.0)$ & - & 5 \\
\hline
\end{tabular}

Table 6c

Overall clinical outcome according to dose of cefepime in the patients

\begin{tabular}{lcccc}
\hline Cefepime use for & Cured & Improved & Failure & Total \\
Treatment of infections & $\mathrm{N}(\%)$ & $\mathrm{N}(\%)$ & $\mathrm{N}(\%)$ & \\
\hline $500 \mathrm{mg}(\mathrm{N}=93)$ & $52(55.9)$ & $19(20.4)$ & $14(15.1)$ & $85(91.4)$ \\
$1 \mathrm{gm}(\mathrm{N}=377)$ & $265(70.3)$ & $90(23.9)$ & $6(1.6)$ & $361(95.8)$ \\
$2 \mathrm{gm}(\mathrm{N}=8)$ & $4(50.0)$ & $4(50.0)$ & - & $8(100)$ \\
\hline
\end{tabular}


Table 6d

Overall clinical outcome according to duration of cefepime therapy in the patients

\begin{tabular}{lcccc}
\hline Duration of therapy & Cured & Improved & Failure & Total \\
& $\mathrm{N}(\%)$ & $\mathrm{N}(\%)$ & $\mathrm{N}(\%)$ & $\mathrm{N}=454$ \\
\hline$<5$ days & $18(62.1)$ & $4(13.8)$ & $7(24.1)$ & $29(6.3)$ \\
$5-7$ days & $255(70.1)$ & $99(27.2)$ & $10(2.7)$ & $364(80.1)$ \\
$>7$ days & $48(78.7)$ & $10(16.4)$ & $3(4.9)$ & $61(13.4)$ \\
\hline
\end{tabular}

According to the physicians' assessment overall tolerability of cefepime was considered as good in more than half of the patients $(56.6 \%)$, excellent in $28.7 \%$, satisfactory in $9.8 \%$ and poor in $4.9 \%$ of patients (Table VII).

\section{Table-VII}

Overall tolerability of cefepime in the patients

\begin{tabular}{lcc}
\hline & \multicolumn{2}{c}{ Patients $(\mathrm{N}=945)$} \\
\cline { 2 - 3 } & $\mathrm{N}$ & $\%$ \\
\hline Excellent & 271 & 28.7 \\
Good & 535 & 56.6 \\
Satisfactory & 93 & 9.8 \\
Poor & 46 & 4.9 \\
\hline
\end{tabular}

No Adverse Events (AE) or Serious Adverse Events were reported in this study by the investigators. Though 25 patients were died and none of the deaths were related to cefepime therapy rather due to congestive heart failure, renal or hepatic failure and multiple organ failure. Nonetheless, the 25 death cases would be considered as Serious Adverse Events. The investigators did not record Adverse Event information in Data Collection Forms designed to collect $\mathrm{AE}$ information although that was requested in the protocol.

\section{Discussion}

This study assessed the effectiveness and tolerability of cefepime among the hospitalized patients in Bangladesh. Bacteriology or culture sensitivity before initiation of antibiotic is not a routine practice in Bangladesh. In this study only 67 out of 1000 patients had bacteriology report at initiation of cefepime therapy. This could also be due to that the majority of the patients were not admitted in intensive care units such as ICU and CCU. Although prescribing cefepime in the absence of a proven or strongly suspected bacterial infection or a prophylactic indication is unlikely to provide benefit to the patient and increases the risk of the development of drug-resistant bacteria. However, empiric use of prophylactic antibiotics is also common here. In this study $52.2 \%$ of patients received cefepime as prophylaxis of the infections related to medical condition or surgical procedure. Cefepime is usually indicated for treatment of pneumonia, bacteremia, uncomplicated or complicated urinary tract infections including pyelonephritis, skin and skin structure infections, intra-abdominal Infections caused by susceptible strains of micro-organisms and also as empiric therapy for febrile neutropenia. In this study cefepime use was in line with prescribing information and used in treatment of lower respiratory tract infections mainly pneumonia, wound infections relevant to fracture, urinary tract infections, septicemia and treating gangrene.

Although, the recommended duration of cefepime therapy is 7 to 10 days, the mean $( \pm S D)$ duration of cefepime therapy in this study was $6.1( \pm 2.1)$ days which was varied from $2(1.7 \%$ of patients) to 14 days (2.1\% of patients). Cefepime therapy decision being physicians' own judgment variations in dose, frequency and duration of therapy was found in this observational study. According to the physicians overall clinical outcome evaluation at end of therapy $52(5.5 \%)$ patients had treatment failure. Therefore, even the treatment was upon the physician decision, short duration of therapy might lead to treatment failure.

In this study $94.4 \%$ of the patients had resolved or improvement in all the signs and symptoms 
of infection at the end of therapy. Similar clinical response rate $(98 \%)$ was observed at post treatment evaluation in hospitalized patients treated with cefepime (Oster et al.). ${ }^{5}$

Generally cefepime is well tolerated. However, few side effects including rash, pruritus, urticaria, fever, headache, nausea, vomiting, diarrhea, dizziness and oral moniliasis may occur in patients. In this study one patient discontinued cefepime therapy due to adverse event and 25 patients died due to congestive heart failure, renal or hepatic failure and multiple organ failure. None of the deaths were related to cefepime therapy yet would be considered as serious adverse events. On physicians' assessment at end of therapy no adverse events was reported in $28.2 \%$ of patients while mild, moderate and serious adverse events were reported in 55.4\%, 8.9\% and $7.4 \%$ of patients respectively. Nevertheless, no adverse event was collected by the physicians as was requested in the study protocol.

In Bangladesh there is no legislation for safety event reporting to health authorities, which led to low awareness on pharmacovigilance among physicians. The investigators participating in studies therefore did not report adverse events or serious adverse events the way it was described in the protocol by the Sponsor. In this study in spite of the safety reporting requirement by the investigators in the Data
Collection Form designed to collect AE information, they did not complete that part. Therefore no AE/SAEs were reported in this study.

\section{Conclusion}

Cefepime therapy in different indications was effective in hospitalized patients for cure (67.5\%) or improvement $(26.9 \%)$ of sign and symptoms. It was also well tolerated in $85.3 \%$ of patients as assessed by the treating physicians

\section{Acknowledgement}

The study was funded by Sanofi Bangladesh Limited.

\section{References}

1. Sheng WH, Wang JT, Chang SC. Efficacy and safety of cefepime in the treatment of serious bacterial infections in hospitalized adult patients. J Microbiol Immunol Infect. 2000 Jun; 33(2): 109-14.

2. Wynd MA, Paladino JA. Cefepime: a fourth-generation parenteral cephalosporin. Ann Pharmacother. 1996 Dec; 30(12): 1414-24.

3. Okamoto MP, Nakahiro RK, Chin A et al. Cefepime: a new fourth-generation cephalosporin. Am J Hosp Pharm. 1994 Feb 15; 51(4): 463-77.

4. Chapman TM, Perry CM. Cefepime: a review of its use in the management of hospitalized patients with pneumonia. Am J Respir Med. 2003; 2(1): 75-107.

5. Oster S, Edelstein H, Cassano K. McCabe R. Open trial of cefepime (BMY 28142) for infections in hospitalized patients. Antimicrobial Agents and Chemotherapy.1990 Jun;34(6): 954-7 